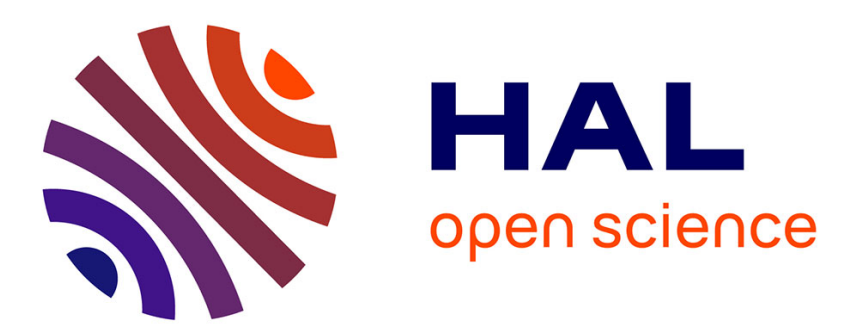

\title{
Multilevel magnetic nanodot arrays with out of plane anisotropy: the role of intra-dot magnetostatic coupling
}

\author{
Vincent Baltz, A. Bollero, B. Rodmacq, B. Dieny, J. P. Jamet, J. Ferré
}

\section{To cite this version:}

Vincent Baltz, A. Bollero, B. Rodmacq, B. Dieny, J. P. Jamet, et al.. Multilevel magnetic nanodot arrays with out of plane anisotropy: the role of intra-dot magnetostatic coupling. European Physical Journal: Applied Physics, 2007, 39, pp.33. 10.1051/epjap:2007107 . hal-01683826

\section{HAL Id: hal-01683826 https://hal.science/hal-01683826}

Submitted on 25 May 2019

HAL is a multi-disciplinary open access archive for the deposit and dissemination of scientific research documents, whether they are published or not. The documents may come from teaching and research institutions in France or abroad, or from public or private research centers.
L'archive ouverte pluridisciplinaire HAL, est destinée au dépôt et à la diffusion de documents scientifiques de niveau recherche, publiés ou non, émanant des établissements d'enseignement et de recherche français ou étrangers, des laboratoires publics ou privés. 


\title{
Multilevel magnetic nanodot arrays with out of plane anisotropy: the role of intra-dot magnetostatic coupling
}

\author{
V. Baltz ${ }^{1, a}$, A. Bollero ${ }^{1, b}$, B. Rodmacq ${ }^{1}$, B. Dieny ${ }^{1}$, J.-P. Jamet ${ }^{2}$, and J. Ferrée \\ 1 DRFMC/SPINTEC (URA CEA/CNRS 2512), CEA Grenoble, 17 Av. Martyrs, 38054 Grenoble Cedex 9, France \\ ${ }^{2}$ Laboratoire de Physique des Solides (UMR CNRS 8502), Université Paris-Sud, 91405 Orsay, France
}

Received: 12 January 2007 / Received in final form: 11 April 2007 / Accepted: 16 May 2007 Published online: 28 June 2007 - (c) EDP Sciences

\begin{abstract}
Intra-dot inter-layer magnetostatic coupling have been investigated in trilayers which consist of two $[\mathrm{Co} / \mathrm{Pt}]$ multilayers with out-of-plane anisotropy and different coercivities separated by a thick $\mathrm{Pt}$ layer. For single domain nanodots, the strong intra-dot inter-layer magnetostatic interactions manifest as a shift of the soft layer hysteresis loop. The amplitude of this shift decreases when the size of the dots is increased and/or when the temperature is increased. Satisfactory agreement is found between calculations and experimental results.
\end{abstract}

PACS. 75 Magnetic properties and materials - 75.70.-i Magnetic properties of thin films, surfaces, and interfaces - 75.60.-d Domain effects, magnetization curves, and hysteresis - 75.60.Ch Domain walls and domain structure - 75.75.+a Magnetic properties of nanostructures

\section{Introduction}

Due to their technological potential for read-heads or magnetic random access memories [1,2], "soft-hard" bilayers $[3-5]$ have been widely studied. Multilevel magnetic recording recently brought a renewed interest for these systems. Indeed, in order to further increase the data storage density [6], it has been proposed that instead of reducing the bit size, one could increase the number of remanent states per cell $[7,8]$. Such a multilevel storage recording can be applied to longitudinal and perpendicular media, either continuous or patterned. A possible way to achieve multilevel media consists in using $N$ magnetic storage layers $(N \geqslant 2)$ with different coercivities. In that case, the recording media might show up to $2^{N}$ stable remanent states. With an appropriate sequence of external applied fields, all the remanent states can be reached. The media can be read with a magnetoresistive read head. Patterned magnetic recording media have also been proposed in order to push further the data storage density towards 1 Terabit in $^{-2}$. The combination of multilevel recording and patterned media has been recently envisaged $[7,8]$. Such systems might be of great technological interest since

\footnotetext{
${ }^{a}$ e-mail: v.a.baltz@leeds.ac.uk

Present address: School of Physics \& Astronomy, University of Leeds, Leeds LS2 9JT, UK.

b Present address: Group of Nanoaerosols, Department of Environment, CIEMAT, Av. Complutense 22, 28040 Madrid, Spain.
}

they could result in a significant enhancement of the media storage densities.

Various magnetic couplings in such multilevel systems can however make the devices susceptible to malfunction. These couplings can have different origins: direct magnetic coupling through pinholes in thin metallic or insulating spacer layer [9], indirect exchange coupling through RKKY interactions [10], orange peel (Néel) magnetostatic coupling in the presence of a correlated roughness at both spacer interfaces [11,12], and finally magnetostatic coupling through stray fields [13]. In the latter case, although these magnetostatic interactions are mostly negligible in uniformly magnetized macroscopic samples, it is no longer the case when the magnetic layers are in a multidomain state, or when the lateral size of the sample is reduced. It has been shown for example in "soft-hard" continuous bilayers with in-plane magnetization that the manipulation of the soft layer can result in an alteration of the magnetic configuration of the hard layer. Peculiar effects resulting from inter-layer magnetostatic $[13-16]$ interactions have also been observed for continuous systems with out-of-plane anisotropy [17-20]. The finite size of the domains accounts for these magnetostatic interactions. It is noteworthy that the smaller the domain size, the larger the magnetic stray fields, and thus the larger the observed detrimental effects. For example, in the case of multilevel recording in patterned media $[7,8]$, we will see that some of the magnetic configurations of the layers can become unstable at zero field, thus lowering the number of remanent states, i.e. of recording levels. 
So far, only few studies on magnetostatic couplings in interacting bilayers have been carried out in systems with out-of-plane anisotropy [17-20] but not yet in related dots arrays. Strong intra-dot inter-layer magnetostatic coupling will actually take place due to the specificity of these systems which combine multilevels, nanoscales and out of plane magnetic anisotropy. We note that since in that case stray fields emanate from the domains themselves, different domain sizes or shapes lead to different stray field amplitudes. In this manuscript we report finite size effects on inter-layer magnetostatic interactions. We have especially studied the magnetization reversal of arrays of nanodots and we have probed some of the effects resulting from intra-dot inter-layer magnetostatic interactions in these single domain multilevel dots, which display high potentials for discrete multilevel recording $[7,8]$.

\section{Sample preparation}

Si wafers were first patterned by electron beam lithography and reactive ion etching [21] to form $0.1 \times 1 \mathrm{~mm}^{2}$ arrays of $\mathrm{Si}$ dots, with lateral sizes ranging from 90 to $400 \mathrm{~nm}$, with a height of $200 \mathrm{~nm}$ and an edge to edge spacing between 50 and $200 \mathrm{~nm}$. In the following, the nanostructures will be noted as follows: (dot size in nanometers)/(edge to edge spacing in nanometers), i.e. $400 \times 100 / 200$ stands for an array of rectangular dots with a surface of $400 \times 100 \mathrm{~nm}^{2}$ and a $200 \mathrm{~nm}$ edge to edge spacing. A scanning electron microscopy image of a $90 \times 90 / 110$ array is shown as an example in Figure $1 . \mathrm{Si} / \mathrm{SiO}_{2} / S_{S} / \mathrm{Pt}(4 \mathrm{~nm}) / S_{H}$ film structure, consisting of a soft $S_{S}$ : $[\operatorname{Pt}(1.8 \mathrm{~nm}) / \mathrm{Co}(0.6 \mathrm{~nm})]_{2}$ and a hard $S_{H}$ : $[\mathrm{Co}(0.6 \mathrm{~nm}) / \mathrm{Pt}(1.8 \mathrm{~nm})]_{4}$ ferromagnetic multilayer stack, separated by a $\mathrm{Pt}(4 \mathrm{~nm})$ spacer layer were DC-magnetron sputtered on these naturally oxidized patterned wafers. The film thereby coats the patterned and surrounding unpatterned (continuous) regions. $S_{H}$ has a larger coercive field since it consists of a larger number of repeats, and also since it is grown on the $4 \mathrm{~nm}$ thick $\mathrm{Pt}$ spacer layer which acts as a buffer. The samples were prepared in a $0.25 \mathrm{~Pa}$ Ar pressure following a base pressure of about $0.4 \times 10^{-5} \mathrm{~Pa}$. The depositions were carried out at room temperature with deposition rates of the order of $0.05 \mathrm{~nm} \mathrm{~s}^{-1}$ and $0.1 \mathrm{~nm} \mathrm{~s}^{-1}$ for cobalt and platinum respectively $[18,21]$. Since the layers are sputtered with a perpendicular-to-plane incidence the deposit covers the top of the pillars (dots) and the inter-dots region (trenches). Given the height of the Si pillars as compared to the thickness of the deposit, the dots and the trenches are magnetically uncoupled $[7,21]$. Indeed, previous Magnetic Force Microscopy (MFM) measurements have revealed that the magnetization of dots with dimensions comparable to ours is independent of the magnetization direction in the trenches [21]. Hysteresis loops were measured at different temperatures, from 4.2 to $450 \mathrm{~K}$ using Polar Magneto-Optical Kerr Effect (PMOKE) setups. The nanostructures (dots and trenches simultaneously) were probed by focusing the laser spot onto the patterned region.

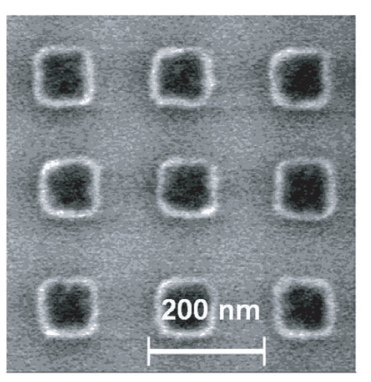

Fig. 1. Scanning electron microscopy image of a $90 \times 90 / 110$ array of nanostructures.

The coupling energy, $J$, due to pinholes, RKKY or orange peel interaction, between two ferromagnetic layers with out-of-plane anisotropy separated by a nonmagnetic spacer decreases roughly exponentially with the non-magnetic spacer thickness [12]. Two Co layers separated by a $1.8 \mathrm{~nm}$ thick $\mathrm{Pt}$ film exhibit a strong ferromagnetic coupling $\left(J>3 \times 10^{-6} \mathrm{~J} \mathrm{~m}^{-2}\right)$, and we can thus consider that $[\mathrm{Co} / \mathrm{Pt}]$ multilayers involving $\mathrm{Pt}$ films of $1.8 \mathrm{~nm}$ are uniformly magnetized. On the other hand, two Co layers separated by a Pt film thicker than $4 \mathrm{~nm}$ exhibit a very small coupling $\left(J<3 \times 10^{-7} \mathrm{~J} \mathrm{~m}^{-2}\right)$. Our $\mathrm{Si} / \mathrm{SiO}_{2} / S_{S} / \mathrm{Pt}(4 \mathrm{~nm}) / S_{H}$ samples can thus be considered as two interacting single magnetic layers. $S_{S}$ and $S_{H}$ will henceforth be designated as layers instead of multilayers.

Our aim is to probe the effects related to intra-dot inter-layer magnetostatic coupling. We have therefore chosen the thinnest $\mathrm{Pt}$ spacer that allows us to minimize RKKY and orange peel coupling and to maximize magnetostatic interactions. It is additionally noteworthy that the geometries of the arrays give rise to densities around few Gdots in ${ }^{-2}$, with inter-dots dipolar coupling of only few tens of Oersted $[20,22,23]$. These values are small (less than $10 \%$ ) compared to the intra-dot magnetostatic interactions that we will further discuss.

\section{Results and discussion}

Figure 2 shows a typical PMOKE hysteresis loop recorded on the nanostructured region for a sample with composition $\mathrm{Si} / \mathrm{SiO}_{2} / S_{S} / \mathrm{Pt}(4 \mathrm{~nm}) / S_{H}$. Two imbricate hysteresis loops with two transitions on each branch, each, can be clearly distinguished. Owing to the vertical incidence of the laser spot during the PMOKE measurements, both the signal coming from the dots and that from the trenches are probed. The two sharp transitions, at relatively low fields (150 Oe and 360 Oe) correspond to the magnetization reversal of the bottom soft layer and top hard layer in the trenches, respectively. The coercive fields obtained for the trenches are slightly larger than those measured on a continuous film (not shown). Such a slight increase of coercitivity accounts for larger nucleation field for the trenches. Given the sharpness of the magnetization reversal in the trenches, it is likely that the base of the silicon dots do not favor domain wall pinning. The two broader 


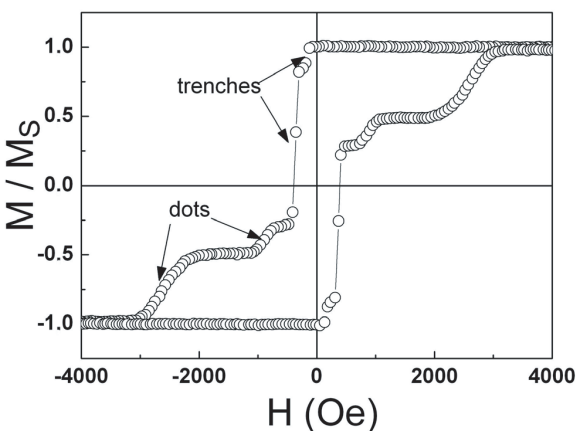

Fig. 2. Room temperature PMOKE hysteresis loop $(\lambda=$ $543.5 \mathrm{~nm}$ ) for a $400 \times 100 / 100$ array of nanostructures with composition $\mathrm{Si} / \mathrm{SiO}_{2} /[\mathrm{Pt} / \mathrm{Co}]_{2} / \mathrm{Pt}(4 \mathrm{~nm}) /[\mathrm{Co} / \mathrm{Pt}]_{4}$.

transitions, at higher fields (900 Oe and 2600 Oe), are ascribed to the magnetization reversal of the bottom soft layer and top hard layer on the dots, respectively. The significant broadening of the transition observed for the dots has been previously reported and attributed to inhomogeneities among the dots which results in a switching field distribution [21]. Larger reversal fields are commonly observed in patterned systems with respect to continuous films or trenches and it can be attributed to the reduced amount of effective nucleation centers for the dots or to a possible change in the magnetization reversal mechanism in comparison to the continuous film $[8,21,24]$. In addition, the edges of the dots play a major role in pinning the magnetization and, in the patterned structures, these edges constitute a more significant part of the structure relative to the total volume and hence lead to an increase of the coercivity. It has to be noted that in both cases, the remanence to saturation ratio remains close to one. Additional MFM images (not shown) allowed us to confirm that in the remanent state, the dots are in an out-of-plane single domain configuration.

Since both the light transmission and reflection corresponding to the bottom and top layers are different, the amplitudes measured by PMOKE cannot be directly linked to the magnetization of the layers [25]. It is also noteworthy that the relative amplitudes between the total signal (bottom soft and top hard layers) from the dots and that from the trenches differ from the nominal ones since the incidence of the laser spot cannot be strictly perpendicular and thus gives rise to shadowing and interferences effects. Varying the optical wavelength $(\lambda)$ of the laser allows tuning the relative amplitude contributions of the dots and trenches. For chosen geometry of arrays and wavelength, the signal coming from the dots or that coming from the trenches can be suppressed. As an example, we show in Figure 3 two limit cases. For a $90 \times 90 / 110$ array, the signal from the trenches is shattered by switching from $\lambda=632.8 \mathrm{~nm}$ to $\lambda=543.5 \mathrm{~nm}$ (Fig. 3a). We note that in this case, the PMOKE coming from the dots and the trenches have opposite signs. In contrast, for a $200 \times 200 / 200$ array, the same change in wavelength results in the suppression of the contribution coming from the dots (Fig. 3b).

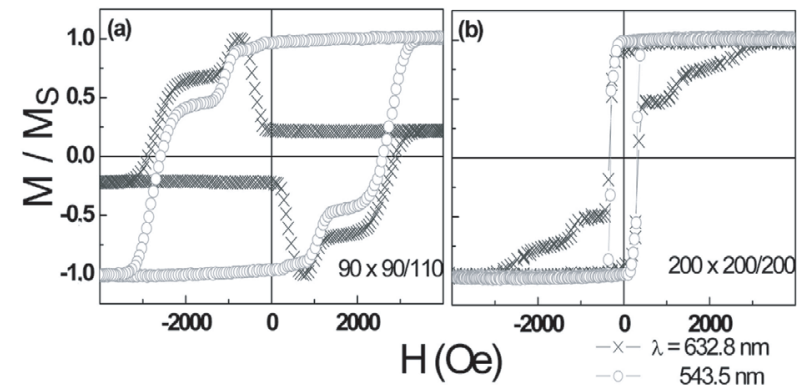

Fig. 3. Room temperature PMOKE hysteresis loop measured at different optical wavelengths $(\lambda): 632.8$ and $543.5 \mathrm{~nm}$, for (a) $90 \times 100 / 110$ and (b) $200 \times 200 / 200$ arrays of nanostructures with composition $\mathrm{Si} / \mathrm{SiO}_{2} /[\mathrm{Pt} / \mathrm{Co}]_{2} / \mathrm{Pt}(4 \mathrm{~nm}) /[\mathrm{Co} / \mathrm{Pt}]_{4}$.
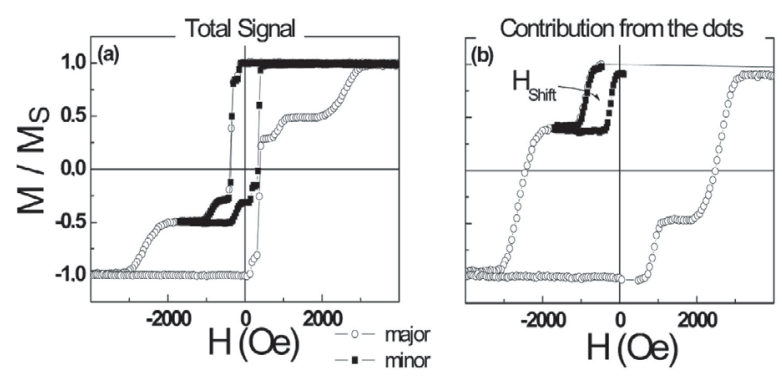

Fig. 4. Major and minor hysteresis loops measured by PMOKE $(\lambda=543.5 \mathrm{~nm})$ at room temperature for a $400 \times 100 / 100$ array of nanostructures with composition $\mathrm{Si} / \mathrm{SiO}_{2} /[\mathrm{Pt} / \mathrm{Co}]_{2} / \mathrm{Pt}(4 \mathrm{~nm}) /[\mathrm{Co} / \mathrm{Pt}]_{4}$. (b) Same loop as in (a) after having artificially removed the contribution from the trenches.

Figure 4a shows a typical major PMOKE hysteresis loop along with a minor hysteresis loop. Only in an attempt to ease the understanding of the loops the contribution from the trenches has been artificially removed. The resulting loops corresponding to the contribution of the dots only, i.e. the contribution of interest is plotted in Figure $4 \mathrm{~b}$. It can be observed that the minor loop corresponding to the bottom soft layer is significantly shifted towards negative magnetic field (from about $550 \mathrm{Oe}$ ), after having saturated the sample in a positive field. This shift corresponds to a ferromagnetic interaction between bottom and top layers. Such a shift is ascribed to strong magnetostatic fields that favor a parallel alignment of the films until the Zeeman energy prevails, like in the case of demagnetized continuous films $[18,20]$. This effect can be destructive in terms of multilevel applications. Indeed, as can be deduced from Figure $4 \mathrm{~b}$, when the shift field is larger than the coercive field of the soft layer, the two intermediate states are no longer stable in zero field. As can be seen in Figure 4b, the minor loop corresponding to the bottom soft layer on the top of the dots is not fully closed. This is ascribed to slight thermal drifts during the measurement. The slope at the beginning of the backward branch of the minor loop is effectively negative which notably drives an initial shift in the normalized magnetization. 


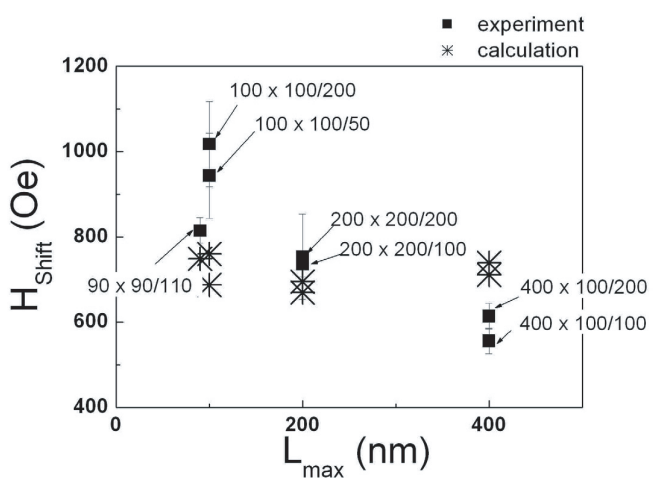

Fig. 5. Dependence of the shift field, $H_{\text {Shift }}$, with the largest lateral dimension, $L_{\max }$, of the dots with composition $\mathrm{Si} / \mathrm{SiO}_{2} /[\mathrm{Pt} / \mathrm{Co}]_{2} / \mathrm{Pt}(4 \mathrm{~nm}) /[\mathrm{Co} / \mathrm{Pt}]_{4}$.

The dependence of the shift field as a function of the lateral dot size is plotted in Figure 5. We note that the value of the shift field is obtained from the difference between the inflexions points of the transitions corresponding to the magnetization reversal of the bottom soft layer deposited on the top of the dots, when measuring the minor loop (see Fig. 4a). This value is directly taken from the uncorrected data (i.e. without subtraction from the signal coming from the trenches). The shift field actually decreases as the dot size increases. Indeed, the stray field created by a finite size domain, e.g. by the hard layer on the soft layer, decreases as its size is increased. It tends to zero in the limit case of an infinite domain.

In order to check for a quantitative agreement between the shift field and the stray field created by the top hard layer on the bottom soft layer, we calculated the variation of the stray fields acting on the soft layer as a function of the dot size. A simplified model which considers facing charged surfaces is used. The derived potential is a function of the cobalt thickness, and the position [26]. The calculated stray fields are those emanating from the top hard layer and sensed at the upper interface of the bottom soft layer (i.e. where their magnitudes are the largest). Typical three-dimensional profiles of such stray fields are plotted in Figure 6 for $90 \times 90 \mathrm{~nm}^{2}$ and $400 \times 100 \mathrm{~nm}^{2}$ dots. The field is maximum at the dots edges, where the spin coordination number is minimum.

Since the hard layer of every dot remains 'up' during the minor loop on the soft layers, the additional inter-dot energy provided through stray fields favours an anti-parallel configuration of the magnetisation of the neighbouring soft layers. This hence competes with the ferromagnetic intra-dot coupling and results in a reduction of the field sensed by a soft layer. We have calculated the inter-dot stray field generated on a central dot by an array of $11 \times 11$ dots pointing 'up' (above 11 dots, the additional inter-dot stray field becomes smaller than 0.5 Oe and can thus be neglected). The maximum inter-dot stray field was obtained for the $100 \times 100 / 50$ array and is around 85 Oe. Note that this value is less than $10 \%$ of the shift field ( 950 Oe).

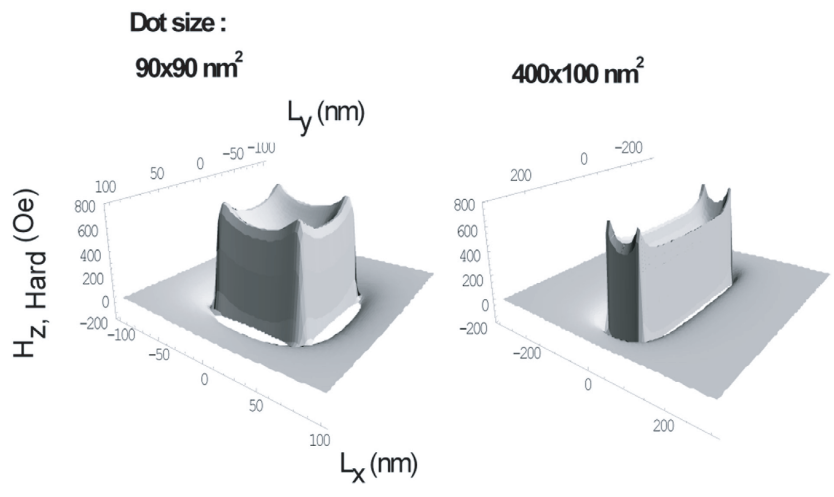

Fig. 6. $3 \mathrm{D}$ mapping of the stray field $H_{Z, H a r d}$ emanating from the hard layer at the lower interface of the soft layer, for $\mathrm{Si} / \mathrm{SiO}_{2} /[\mathrm{Pt} / \mathrm{Co}]_{2} / \mathrm{Pt}(4 \mathrm{~nm}) /[\mathrm{Co} / \mathrm{Pt}]_{4} \operatorname{dots}$.

The maximum value of the calculated stray field created by the top hard layer at the upper interface of the bottom soft layer (Fig. 6), from which we subtracted the calculated inter-dot stray field, is plotted in Figure 5, along with the experimental data. The calculations provide the right order of magnitude of the shift field, hence confirming that the hysteresis loop shift can be directly ascribed to strong inter-layer magnetostatic interactions. From Figure 5 the shift field seems to be larger as the dimension between the dots is increased. Indeed, the closer the dots, the larger this inter-dot coupling and the smaller the shift field. We note that the magnetization reversal process presumably involves complicated nucleation events that cannot all be taken into account by our simplified calculations, which thus do not accurately account for the size dependence of the shift field.

The thermal dependences of the saturation magnetization of the dots and of the shift field, as obtained from PMOKE measurements, are plotted in Figure 7. As mentioned above, a good choice of the optical wavelength allowed us to eliminate the PMOKE signal coming from the trenches over a wide range of temperatures $(4.2 \mathrm{~K}<T<300 \mathrm{~K})$ [25]. In contrast, our high temperature $(300$ to $450 \mathrm{~K})$ set-up only works at a fixed optical wavelength. The four transitions ascribed to the magnetization reversal of the soft and hard layers in the trenches and on the dots are thus probed. We could distinguish the signal corresponding to the dots with respect to the signal corresponding to the trenches. Given the weak signal from the dots by comparison with that from the trenches at this given optical wavelength, it was not possible to differentiate clearly the signal from the hard layer and that from the soft one on the dots. Therefore and for consistency, only the sum of both signals coming from the dots is plotted in Figure 7a, over the whole range of temperatures studied. A full set of measurements on continuous films with the same compositions (inset of Fig. 7a) also revealed that both the soft and the hard layer display similar thermal dependences of the magnetization over a large range of temperatures (from around 100 to $400 \mathrm{~K}$ ). This result is not surprising since the cobalt and platinum sublayers of 

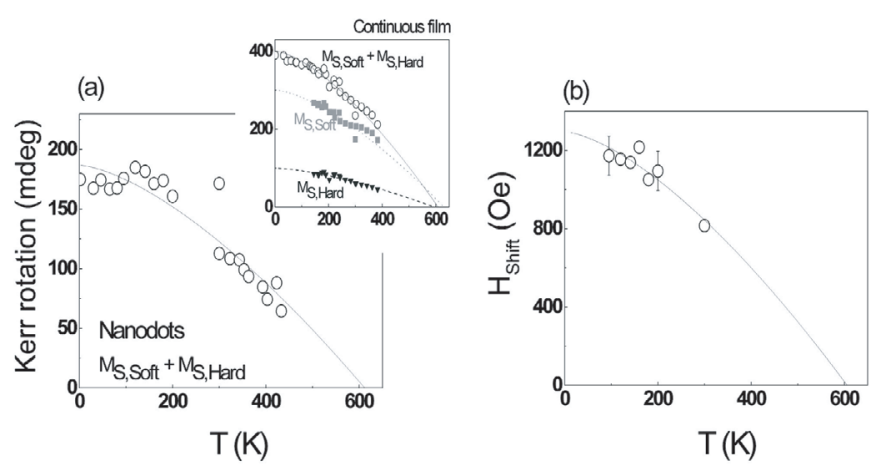

Fig. 7. (a) Temperature dependence of the Kerr rotation of the soft layer, $M_{s, \text { Soft }}$, added to that of the hard layer, $M_{s, \text { Hard }}$, for $90 \times 90 / 110$ dots with composition $\mathrm{Si} / \mathrm{SiO}_{2} /[\mathrm{Pt} / \mathrm{Co}]_{2} / \mathrm{Pt}(4 \mathrm{~nm}) /[\mathrm{Co} / \mathrm{Pt}]_{4}$. For comparison, the inset shows the temperature dependences of $M_{s, \text { Soft }}$ and $M_{s, \text { Hard }}$ for the continuous film. (b) Temperature dependence of the shift field, $H_{S h i f t}$, extracted from PMOKE measurements. The lines correspond to $T^{3 / 2}$ fits.

the soft and hard 'layers' are of the same thickness $(0.6 \mathrm{~nm}$ for the cobalt sublayer and $1.8 \mathrm{~nm}$ for the platinum sublayer).

As shown in Figure 7, the dependences of both the saturation magnetization of the layers and the shift field with the temperature are similar. We note that the shift field could only be extracted when using our low temperature setup and above $100 \mathrm{~K}$. Below $100 \mathrm{~K}$ coercive field distribution overlapping between the soft and hard layers on the dots did not allow us to clearly distinguish the respective transitions. Knowing that the stray field created by a layer is proportional to its saturation magnetization, $M_{S}$, the comparison of the thermal behaviors of $M_{S}$ and the shift field allowed us to further confirm the direct correspondence between stray and shift fields. The dependences shown in Figure 7 can both be approximated by a $\left(1-B \cdot T^{3 / 2}\right)$ Bloch law [27] (i.e. based on thermal excitation of magnons). We note that the Bloch law is not universal [28]. For example some authors report other behaviors for very low temperatures [29].

From the $\left(1-B \cdot T^{3 / 2}\right)$ fits, we deduce a Curie temperature around $600 \mathrm{~K}$, in good agreement with that previously deduced for continuous films [20], and a slope $B$ of the order of $6.7 \times 10^{-5} \mathrm{~K}^{-3 / 2}$. From this parameter, we calculate an exchange stiffness of around $2.6 \times 10^{-7} \mathrm{erg} \mathrm{cm}^{-1}$ which is also in good agreement with previous results [20]. Both the Curie temperature and the exchange stiffness are about half the values reported for bulk cobalt. This accounts for a change in atomic environment in agreement with previous studies reported in the literature [30].

Considering further applications, the thermal dependence of the shift field has to be taken into account for the implementation of multilevel patterned media, since any significant variation of the shift field in real devices could lead to malfunction.

\section{Conclusion}

To conclude, we have studied multilevel patterned nanodots with out-of-plane magnetic anisotropy and fabricated by a pre-patterning method. These systems combine the advantages inherent to both multilevel and patterned media and might be of great technological interest since they could result in a significant enhancement of the media storage densities. Strong magnetic stray fields resulting from the finite size of the domains in systems with out-of-plane anisotropy can lead to large ferromagnetic inter-layer coupling in trilayers which consist of two ferromagnetic layers separated by a non-magnetic layer. In the present single domain nanostructures, this leads to a shift of the soft layer hysteresis loop which decreases when increasing the size of the nanostructures or the temperature. Such intra-dot couplings will play a negative role with regards to the stability of the stored data and have to be taken into account, and possibly compensated, for the implementation of multilevel patterned media, in order to stabilize all the levels at remanence.

The authors acknowledge S. Landis from the CEA/LETI/D2NT for the fabrication of the pre-patterned silicon substrates; A. Mougin from the LPS Orsay for her precious help; and A.T. Hindmarch from the EC Stoner Laboratory of the University of Leeds for the critical reading of the manuscript. A. Bollero acknowledges funding from the NEXBIAS RTN (Grant No. HPRN-CT-2002-00296).

\section{References}

1. B. Dieny, in Magnetoelectronics (Elsevier Academic Press, Amsterdam, 2004), p. 67

2. M.G. Samant, S.S.P. Parkin, Vacuum 74, 705 (2004)

3. M.N. Baibich, J.M. Broto, A. Fert, F. Nguyen Van Dau, F. Petroff, P. Etienne, G. Creuzet, A. Friederich, J. Chazelas, Phys. Rev. Lett. 61, 2472 (1988)

4. G. Binasch, P. Grünberg, F. Saurenbach, W. Zinn, Phys. Rev. B 39, 4828 (1989)

5. S.S.P. Parkin, N. More, K.P. Roche, Phys. Rev. Lett. 64, $2304(1990)$

6. A. Moser, K. Takano, D.T. Margulies, M. Albrecht, Y. Sonobe, Y. Ikeda, S. Sun, E.E. Fullerton, J. Phys. D 35 (2002) 157

7. V. Baltz, S. Landis, B. Rodmacq, B. Dieny, J. Magn. Magn. Mat. 290-291, 1286 (2005)

8. M. Albrecht, G. Hu, A. Moser, O. Hellwig, B.D. Terris, J. Appl. Phys. 97, 103910 (2005)

9. J. Wang, Y. Liu, P.P. Freitas, E. Snoeck, J.L. Martins, J. Appl. Phys. 93, 8367 (2003)

10. O. Hellwig, T.L. Kirk, J.B. Kortright, A. Berger, E.E. Fullerton, Nat. Mater. 2, 112 (2003)

11. L. Néel, C.R. Hebd, Seances Acad. Sci. 255, 1676 (1962)

12. J. Moritz, F. Garcia, J.C. Toussaint, B. Dieny, J.P. Nozières, Europhys. Lett. 65, 123 (2004)

13. L. Thomas, M.G. Samant, S.S.P. Parkin, Phys. Rev. Lett. 84, $1816(2000)$

14. C. Cowache, B. Dieny, S. Auffret, M. Cartier, R.H. Taylor, R. O'Barr, S.Y. Yamamoto, IEEE Trans. Magn. 34, 843 (1998) 
15. W.S. Lew, S.P. Li, L. Lopez-Diaz, D.C. Hatton, J.A.C. Bland, Phys. Rev. Lett. 90, 217201 (2003)

16. W. Kuch, L.I. Chelaru, K. Fukumoto, F. Porrati, F. Offi, M. Kotsugi, J. Kirschner, Phys. Rev. B 67, 214403 (2003)

17. H.W. Fuller, D.L. Sullivan, J. Appl. Phys. 33, 1063 (1962)

18. B. Rodmacq, V. Baltz, B. Dieny, Phys. Rev. B 73, 092405 (2006)

19. S. Wiebel, J.-P. Jamet, N. Vernier, A. Mougin, J. Ferré, V. Baltz, B. Rodmacq, B. Dieny, Appl. Phys. Lett. 86 142502 (2005); S. Wiebel, J.-P. Jamet, N. Vernier, A. Mougin, J. Ferré, V. Baltz, B. Rodmacq, B. Dieny, J. Appl. Phys. 100, 043912 (2006)

20. V. Baltz, A. Marty, B. Rodmacq, B. Dieny, Phys. Rev. B 75, 014406 (2007); V. Baltz, Effets de taille finie sur les couplages magnétostatiques et l'anisotropie d'échange dans le domaine de l'enregistrement magnétique, thèse de l'Université Joseph Fourier de Grenoble (2005), and references therein

21. S. Landis, B. Rodmacq, B. Dieny, Phys. Rev. B 62, 12271 (2000); S. Landis, Réseaux de plots submicroniques réalisés à partir de substrats pré-gravés, thèse de l'Université Joseph Fourier de Grenoble (2001), and references therein
22. T. Aign, P. Meyer, S. Lemerle, J.P. Jamet, J. Ferré, V. Mathet, C. Chappert, J. Gierak, C. Vieu, F. Rousseaux, H. Launois, H. Bernas, Phys. Rev. Lett. 81, 5656 (1998)

23. V. Repain, J.P. Jamet, N. Vernier, M. Bauer, J. Ferré, C. Chappert, J. Gierak, D. Mailly, J. Appl. Phys. 95, 2614 (2004)

24. J.-P. Jamet, S. Lemerle, P. Meyer, J. Ferré, B. Bartenlian, N. Nardou, C. Chappert, P. Veillet, F. Rousseaux, D. Decanini, H. Launois, Phys. Rev. B 57, 14320 (1998)

25. J. Ferré, Linear and non linear magneto-optical effects: magnetism of thin film structures, in Magnetism and Synchrotron Radiation, Lecture Notes in Physics, edited by E. Beaurepaire, F. Scheurer, G. Krill, J.-P. Kappler (Springer Verlag, 2001)

26. A. Hubert, R. Schäfer, Magnetic Domains (Springer, 1998)

27. C. Kittel, Solid State Physics (Dunod, 1998), p. 409

28. S.J. Yuan, L. Sun, H. Sang, J. Du, S.M. Zhou, Phys. Rev. B 68, 134443 (2003)

29. J.F. Bobo, L. Gabillet, M. Bibes, J. Phys. Cond. Mat. 16, S471 (2004)

30. E. Della Torre, L.H. Bennet, R.E. Watson, Phys. Rev. Lett. 94, $147210(2005)$ 